\title{
Linking optical and infrared observations with gravitational wave sources through variability
}

\author{
C W Stubbs \\ Department of Physics \\ Department of Astronomy \\ Harvard University \\ 17 Oxford Street \\ Cambridge MA 02138 USA \\ E-mail: stubbs@physics.harvard.edu
}

\begin{abstract}
Optical and infrared observations have thus far detected more celestial cataclysms than have been seen in gravity waves $(\mathrm{GW})$. This argues that we should search for gravity wave signatures that correspond to flux variability seen at optical wavelengths, at precisely known positions. There is an unknown time delay between the optical and gravitational transient, but knowing the source location precisely specifies the corresponding time delays across the gravitational antenna network as a function of the GW-to-optical arrival time difference. Optical searches should detect virtually all supernovae that are plausible gravitational radiation sources. The transient optical signature expected from merging compact objects is not as well understood, but there are good reasons to expect detectable transient optical/IR emission from most of these sources as well. The next generation of deep wide-field surveys (for example PanSTARRS and LSST) will be sensitive to subtle optical variability, but we need to fill the "blind spots" that exist in the Galactic plane, and for optically bright transient sources. In particular, a Galactic plane variability survey at $\lambda \sim 2 \mu \mathrm{m}$ seems worthwhile. Science would benefit from closer coordination between the various optical survey projects and the gravity wave community.

PACS numbers: 95.55.Ym, 95.75.Wx, 95.80.+p
\end{abstract}




\section{Introduction, and some assertions}

This paper addresses the pragmatic challenges of linking optical and infrared observations with the time series recorded by the worldwide network of gravity wave antennae. This is particularly interesting as the optical astronomy community is moving towards an era of "celestial cinematography" where the entire accessible sky will be imaged to unprecedented depth, with near-real-time detection and classification of variable sources.

I will start with some assertions:

(i) More celestial transient and variable sources have been seen to date at optical wavelengths than have been seen in gravity waves. These detections include a diversity of supernovae, QSO's to very remote distances, and the optical counterparts to GRB's.

(ii) An uncoordinated patchwork of optical surveys are monitoring the sky at different revisit cadences, passbands, and flux levels. The next generation of surveys (such as PanSTARRS and LSST) will be sensitive to very subtle changes in flux, for sources with variability timescales of more than a few days, across most of the sky. We will soon start building an all-sky catalog of variability down to $22^{\text {nd }}$ magnitude.

(iii) In the context of potentially detectable gravity wave (GW) sources, optical monitoring currently has two "blind spots", one in flux and the other in sky coverage. A core collapse supernova in the local group is (in my opinion) more likely to be first detected by eye than by a camera system; we are biased against detecting bright sources in our local neighbourhood, as they would rapidly saturate the dynamic range of most survey systems. Similarly, the monitoring across the disk of our own Milky Way is incomplete. LSST, for example, is considering avoiding the region with Galactic latitude $|b|<20$, due to source confusion.

\section{Exploiting optical variability to trigger a constrained GW analysis}

These considerations suggest that (at least in the pre-LISA era) we should reverse the paradigm in which the gravitational wave observatories would, upon detection of a burst signal, pick up a red telephone and call the optical hotline, 1-(800)-CHASEIT. Instead, it seems that the expected sources of interest have variability at optical wavelengths that is more readily detectable than the associated gravity waves. This approach can of course be combined with making optical followup observations of regions on the sky that correspond to source directions from low-significance GW triggers.

Using frame subtraction techniques (e.g. Barris et al. 2005, Miknaitis et al. 2007)

the astronomical community has achieved essentially Poisson-limited sensitivity to optical transients. Implementing pipelined image processing on cluster computers has allowed same-day detection and classification of optical transients.

The main point of this paper is that we should exploit optical variability to search for gravity waves, in addition to the other way around. In a sense this amounts to 
making numerous "pointed" observations with the current generation of gravity wave detectors. I advocate a program in which detected variability of interest at optical and infrared wavelengths be used as a prior for the analysis of the time series data from the network of gravity wave antennae. This approach can of course augment and complement "isotropic" burst detection algorithms that ignore known variable optical sources, but the advantage of searching for signals from a known direction is that the time delays and amplitude ratios of the detected strain signals can presumably be used suppress the false detection rate. Furthermore, as was recently shown (LIGO \& Hurley (2007)) for a GRB source, even an upper bound from the gravity wave detectors can be used to set limits, in SI units, on the time rate of change of the mass quadrupole moment of the event. There is considerable merit in finding ways to exploit the upper limits to gravitational emission from celestial fireworks, at known distances, seen in the optical. Finally, one could imagine stacking, with appropriate time differences based on various source directions, the different antenna time series and then running a transient detection algorithm as a function of the time delay between the optical and gravitational transient. (I thank Bence Kocsis for making the stacking suggestion.)

Making an association of GW sources with optical and IR data will greatly facilitate both the fundamental physics exploitation and the astrophysical understanding of these systems. Another major goal in associating gravity wave sources with optical emission is obtaining a GW-independent redshift of and (perhaps) distance to the source. This will be important in assessing systematics when constructing a Hubble diagram with gravity wave sources (Holtz \& Hughes (2005); Deffayet \& Menou, (2007)).

There are many stars and galaxies on the sky. By restricting our consideration for GW linkage to those objects that exhibit appropriate variability and that lie within the likely GW detection range we can greatly reduce the surface density of candidate optical counterparts. This presumes that gravity wave emission is frequently associated with optical variability. I argue below that this likely true.

\subsection{An analysis strategy for seeking $G W$ transients from a fixed location}

The positional uncertainties for optical variability are subarcsecond. This is to be compared with many arcminutes to degrees of expected positional uncertainty from even robust gravity wave detections (Sylvestre (2003)). This makes the optical-togravitational route considerably easier than the converse. Knowing the position of the candidate GW source on the sky to an angular uncertainty $\sigma_{\phi}$ of an arcsecond

specifies the time delays across the antenna network to a typical precision of $\sigma_{t} \sim$ $\sigma_{\phi}(B / c) \cos (\phi(t))$ where $\mathrm{B}$ is the baseline separation between the antennae, $\phi(t)$ is the angle between $\vec{B}$ and the direction to the source, and $c$ is the speed of light. For the two LIGO detectors with a separation of $B=3000 \mathrm{~km}$ this amounts to a typical uncertainty $\sigma_{t}$ of tens of nanoseconds. The limited bandwidth of the GW antennae preclude taking full advantage of this, of course.

The GW search can be carried out by requiring joint excursions in strain at the 
various antennae in the network with a tightly constrained arrival time difference, but at an unknown arrival time (since we don't know the time delay between the optical and GW transients). The known location specifies a unique relationship between the arrival time difference(s) and the time delay between the optical and the GW transient, as the Earth rotates relative to the fixed source location.

\subsection{Optical variability from core collapse supernovae}

Core collapse supernovae do have intense transient optical emission. Typical B-band absolute magnitudes for type II supernovae are (Richardson et al. (2002)) $\mathrm{M}_{B}^{S N I I} \sim-18$. A detonation of such an object in M31, at a distance modulus of $\mu=24.3$, would have a peak apparent magnitude of around $B=-18+24.3 \sim 6$. By the standards of modern optical astronomy this is a really bright object! A detonation in the Milky Way, even in heavily obscured regions near the Galactic center, would likely be even brighter. As discussed below, these objects would saturate most current sky surveys, except at the very beginning and end of their light curve. More exotic objects, such as supernova 2006gy, which is thought to arise from the collapse of a very massive star (Smith et al. 2006), are even brighter in the optical.

We see more of these objects at optical wavelengths than in gravity waves or

neutrinos. Section 4 describes the numerous spectrally confirmed type II supernovae that were detected on the sky during the S5 run.

It seems safe to assume that any core collapse SN that could produce a detectable gravitational signal would have a huge, and readily detectable, variable optical signature. Since the current estimates (e.g. Ott et al. (2006)) for the strength of the SN gravity wave emission indicate LIGO only being able to see normal core collapse supernovae if they occur within the local group, the challenge at optical wavelengths is fielding a system that would not immediately saturate. Given that the incidence of core collapse supernovae within the local group is rare, it seems sensible to field the relatively inexpensive instrumentation that would generate precise early multiband light curves of such an event.

\subsection{Optical variability from coalescing compact objects}

The mergers of compact objects are considered a likely source of detectable gravitational radiation. Ground-based interferometers and LISA have different spectral regimes of optimal sensitivity, and therefore the compact object masses of interest are different for the two detector types. A variety of mechanisms have been considered for producing electromagnetic emission in association with mergers. An illustrative sampling of these mechanisms includes:

- Optical emission powered by radioactive ejecta, as happens in SN Ia's (Rosswog $(2005))$, 
- Conversion of gravitational radiation into electromagnetic waves through interactions in the local circumstellar environment (Sylvestre(2003)), and

- Gravitational radiation from black hole ringdown converting to electromagnetic radiation through local magnetic fields (Clarkson et al. (2004)).

An open question is what proportion of gravity-wave-detectable coalescence sources have undetectably low associated optical variability. As we push the optical limits into the regime of $24^{\text {th }}$ magnitude, I surmise that the optically silent fraction is low. Even in the case of $\mathrm{BH}-\mathrm{BH}$ mergers, the complex interaction between the emitted gravity waves, the surrounding material, and any local magnetic fields could well produce detectable optical/IR emission. If we take the extreme case where the $\mathrm{BH}-\mathrm{BH}$ events have no detectable associated optical transient, we'd only miss perhaps one third of the total merger events (see Table 2 of Belcyznski et al. (2002)) by triggering on optical emission.

\subsection{The LIGO regime: mergers of 1 to 100 solar mass compact objects}

Sylvestre (2003) estimated the optical emission for compact object mergers in the LIGO band. Typical optical magnitudes for NS-NS mergers were estimated as bring brighter than $\mathrm{R}=20$, even out to distances of hundreds of Mpc. One mechanism described in that paper was expected to produce an optical transient that lasted about a day. This argues in favor of an optical wavelength sky survey that monitors the entire observable sky down to $20^{\text {th }}$ magnitude, daily.

\subsection{The LISA regime: merging supermassive black holes}

A number of authors (e.g. Armitage \& Natarajan (2002); Milosavljevic \& Phinney (2006); Bogdanovic et al. (2007), Dotti et al. (2006)) have explored the electromagnetic emission that might accompany the merger of compact objects in the mass range $\left(10^{5}\right.$ $10^{7}$ solar masses) that would produce GW signals in the spectral region where LISA is sensitive. These predictions for electromagnetic LISA counterparts exhibit considerable variation.

High mass BH-BH mergers $\left(10^{5}-10^{7}\right.$ solar masses) are expected to exhibit a substantial change in their Xray spectra, and some of this radiation may be re-processed into the OIR regime if the optical depths around the source are high enough. The gravitational and electromagnetic transients are expected to coincide within a $\Delta t$ of order a few years. Milosavljevic and Phinney (2006) also refer to what they call a "weak" prompt electromagnetic transient that is simultaneous with the merger event, but they did not provide a quantitative estimate of this signature.

Rosswog (2005) performed numerical simulations of NS-BH mergers with small mass

ratios, and found the ejection of substantial amounts of radioactive material that powers an electromagnetic transient comparable to that of a type Ia supernova. Armitage \& Natarajan (2002) considered coalescing objects embedded in an accretion disk. 
There appears to be a rich phenomenology surrounding the merger process for two supermassive black holes, and as yet no strong consensus on the likely optical/IR emission from such systems.

Regarding the identification of optical counterparts of LISA sources, where we expect there to be numerous detections, a number of workers have considered the challenge of making the linkage in the GW-to-optical direction. For example Kocsis et al. (2006) have considered the 3-d density of QSO's that might correspond to LISA sources.

Given the attention that is already being given to this approach in the LISA era, the focus in this paper is making the connection starting from optical detections, in the sparse-detection regime. We therefore need to review the various optical transient detection opportunities that exist and are on the horizon, and the sky density of variable sources.

\subsection{The Sky Density of Anomalous Variable Objects}

At $\mathrm{R} \sim 21$ there are a few thousand galaxies per square degree. The stellar density depends on Galactic latitude and longitude. If position on the sky is the only metric used to establish a linkage to an observed object, for GW positional uncertainties of order a degree there is more than a thousand-fold ambiguity in identifying the optical counterpart. An estimate of GW sensitivity vs. distance can be used in conjunction with photometric redshifts or some other estimator of distance to reduce the catalog of potential host galaxies, but variability provides important additional information.

Becker et al. (2004) provide insights into the variable sky at faint flux levels, and that paper also provides a good overview of variability surveys. They conducted a search for faint transients. Their upper limit (95\% confidence) on variability on time scales of 1900 seconds in the range $19.5<\mathrm{R}<23.4 \mathrm{mag}$ is 5.3 events $\mathrm{deg}^{-2} \mathrm{day}^{-1}$. They detected three short duration transients in the $\mathrm{B}$ band, one of which is identified as a flare in a Galactic star.

Data from the SDSS survey show (Sesar el al. (2007)) that only about $2 \%$ of the point sources on the sky with $g<20.5$ exhibit fractional flux variations of greater than 5\%. At $g \sim 20$ quasars comprise nearly two thirds of the photometrically variable sources. A combination of temporal variability, magnitude, and color information can do a good job of distinguishing between variable Galactic stars and extragalactic sources.

The majority of the photometric variables can be readily classified into known bins of astronomical taxonomy. For our purposes this can be used to great advantage. For example, little GW emission is expected from RR Lyra and Cepheid variable stars. If we imagine that perhaps $1 \%$ of the variables exhibit anomalous behavior so as to be plausible transient GW counterparts, then their density the sky is a fraction of around $10^{-4}$ of the static sources to $\mathrm{R}=20$, or a few per square degree, which is a viable regime. Whether optical transients trigger GW searches, or whether the goal is to go in the other direction and associate optical sources with GW triggers, concentrating on anomalous 
variable sources will ease the task.

\section{A diversity of optical survey systems}

There are a number of options when scanning the skies for optical variability that might have associated gravitational wave emission. For a fixed detector area, in square $\mathrm{cm}$, the focal length of the optical system determines the field of view (a solid angle $\Omega$ ). There is a practical limit to how fast the optical system can be, and so for a fixed detector area a larger aperture optical system necessarily has a smaller angular field of view. The etendue of the overall system, the $\pi R^{2} \Omega$ product of collecting area times field of view, is an important figure of merit for a survey system. The faintest source that can be detected, however, depends only on the collecting area and not on the field of view. Table 1 presents the relevant properties of some representative optical survey systems, and illustrative examples are described in the subsections that follow.

\subsection{All-sky cameras}

At one extreme are instruments that use a fisheye lens to monitor the entire accessible sky at once. Due to the optical constraints described earlier, these systems have very small effective apertures. A nice example of implementing this approach is the ConCam system (Shamir \& Nemiroff (2005)), which has now been installed at multiple observatories. The sensitivity of these systems is comparable to that of the naked eye, around $6^{\text {th }}$ magnitude, and they are usually pointed at the zenith and left to run unattended.

\subsection{All-sky surveys}

A system optimized for reaching fainter magnitudes, but also with full sky coverage, is the All Sky Automated Survey (ASAS) (Paczynki, 2006). ASAS was designed to detect variability across the entire sky, down to $14^{\text {th }}$ magnitude, capturing an image of each patch of sky with daily revisits. The field of view of ASAS allows a succession of images to cover the accessible sky once per night. An ambitious set of next-generation sky surveys, yet to enter full operation, are discussed below.

\subsection{GRB followup systems}

A suite of instruments was designed to make rapid optical followup observations of GRB's. This includes the ROTSE series of devices (Yost et al. (2006)), the WASP instrument (Pollacco et al. (2006)), and Raptor (Wozniak et al. (2005)). The field of view of these systems was initially chosen to correspond to the expected location uncertainty from BATSE, around 15 degrees, but subsequent improvements in GRB

locations have led to smaller fields and correspondingly larger apertures. Some of these groups are now contemplating or are undertaking sky surveys with these instruments. 


\subsection{Killer asteroid, planetary transit, and microlensing surveys}

These are time domain surveys, usually over a limited portion of the sky. Surveys designed to detect potentially hazardous asteroids are typically implemented on $1 \mathrm{~m}$ class telescopes, in order to attain sensitivity to asteroids smaller than $1 \mathrm{~km}$ in size. This reduces the field of view, and so these systems usually concentrate their observations in the plane of the ecliptic, often avoiding regions of low Galactic latitude. One of the more successful such systems is LINEAR (Stokes et al. (1998)). The asteroid surveys typically take re-observations of a field about a half hour after the night's initial image.

Transit surveys take multiple images of fields centered on relatively bright (to allow high resolution spectroscopic radial velocity followup) stars in order to search for the photometric signature of a transiting planet. While these projects reach impressive levels of sensitivity to flux changes from the target star, their sky coverage is typically insufficient to be of interest in the context of gravity wave counterparts.

Another example of time domain systems that survey a limited region on the sky

are the microlensing search systems. Of the telescope systems that were originally specifically designed to carry out microlensing surveys, one (MACHO) was lost to an Australian brushfire, one (EROS) is no longer in operation, and one (OGLE) continues to monitor fields in the Magellanic Clouds and fields in the Galactic plane.

\subsection{Dedicated supernova searches}

A relatively small field of view (perhaps 10 arcminutes across) can be used to monitor specific nearby galaxies. This is the path taken by the KAIT supernova system (Filippenko et al. (2001)), sited at Lick Observatory. This telescope runs through a list of target galaxies, and the successive images are searched for the transient brightening of a point source that is characteristic of a supernova.

\subsection{Multipurpose telescopes}

The Sloan Digital Sky Survey (SDSS) has both an imaging and a spectroscopic mode. A portion of the time on the SDSS telescope has been used to do repeated imaging (Sessar et al. (2007)) on one particular region, SDSS stripe 82. SDSS is an example of a system that has good area coverage capability, but other scientific priorities (including spectroscopy) limit the time spent on time domain imaging, such that high cadence allsky imaging is not currently being undertaken. Other telescopes with wide field cameras (e.g. CFHT, SUBARU, and the NOAO 4 meter telescopes at Kitt Peak and CTIO) are allocated to a diversity of projects, and this limits their spending a large fraction of the telescope time for a multi-epoch sky survey.

\subsection{The next generation: PanSTARRS, SkyMapper, VST, and the LSST}

The astronomical community is now building multiple wide field sky survey systems. These include PanSTARRS, on the Haleakala volcano on the island of Maui, SkyMapper, 
destined for the Siding Spring Observatory in Australia, the VLT Survey Telescope (VST) in Chile, and the Large Synoptic Survey Telescope (LSST), also destined for construction in Chile. PanSTARRS-1, VST, and SkyMapper are all slated to start science observations sometime in 2008. Of particular interest in the context of gravitational wave sources, the PanSTARRS-1 system has an auxiliary imager, the Imaging Sky Probe (ISP), that uses a single imager and a small aperture fore-optic that is co-boresighted with the main instrument to extend the bright end of the dynamic range up to brighter than $\sim 8^{t h}$ magnitude. This ISP will be operated in tandem with the main imager, mainly for calibration purposes. The VST system is not dedicated to a single integrated survey strategy. The LSST is the most ambitious of these systems, and current plans call for it to begin operation in 2014. Both LSST and the multi-aperture version of PanSTARRS (PS-4) are currently "unencumbered by full funding".

While these systems will jointly provide variability detection down to very faint flux levels, the different projects have different goals for sky coverage. For example at the present time the LSST default plan avoids observing in regions of low Galactic latitude, due to source confusion. It is important to recognize that as these new survey systems come online, what is now rare will become commonplace, and we will catch glimpses of processes that are presently unobservably infrequent.

The PanSTARRS-1 telescope is expected to detect hundreds of supernovae per month, and dozens of these are expected to be brighter than $\mathrm{R}=20$. Among the imminent next-generation systems, PanSTARRS-1 (PS-1) is unique in its goal of realtime detection of transient phenomena at faint levels, across the entire observable sky. In particular PS-1 is well suited to detecting faint optical "orphan afterglows" that might accompany compact object mergers, but that beam their gamma rays away from us. Zou et al. (2007) estimate that a typical afterglow should be brighter than $\mathrm{R}=21$ for many days. According to their Figure 3 we might expect PS-1 (which revisits each patch of $3 \pi$ sr often enough to not miss one) to see one such source at any given time.

\subsection{What about the infrared?}

The discussion here emphasizes optical surveys, taken to be the wavelength region over which silicon CCDs are sensitive, longward of the ozone cutoff: $350 \mathrm{~nm}<\lambda<1000$ $\mathrm{nm}$. There is merit in considering wide-field surveys at longer wavelengths, particularly towards the obscured regions near the Galactic center. Lucas et al. (2007) describe the UKIDSS Galactic Plane Survey in the J,H,K bands, but I am not aware of a program to do variability monitoring using the IR across the Galactic plane. It is well worth considering undertaking an IR Galactic variability survey to augment the gravitational wave searches. If the coalescing compact object mergers are enshrouded in and/or behind optically thick material, IR variability would be a useful way to identify potential GW sources even through heavy attenuation in the optical.

A typical core collapse SN has an IR peak magnitude of $K \sim-18$ (Grossan et al. (1999)). Table 2 illustrates the stunning difference in transmission through "dust" in 
Table 1. Some Optical Sky Surveys. This is a non-exhaustive list of existing and planned $\left(^{*}\right)$ optical sky surveys. One figure of merit for a survey is the product of collecting area times its field of view (FOV), the A $\Omega$ product. Another figure of merit is the source intensity to which it is sensitive, which is primarily a function of only aperture. The limiting point source magnitudes listed in the final column are very approximate, meant to illustrate the rough range of sensitivities. Similarly, the $A \Omega$ products ignore obscuration in the optics, but do give a rough basis for comparison between systems. An interesting comparison is that the Hubble Space Telescope has $\mathrm{A} \Omega=0.016(\mathrm{mdeg})^{2}$.

\begin{tabular}{lccccc}
\hline System & $\begin{array}{c}\text { Diam. } \\
(\mathrm{m})\end{array}$ & $\begin{array}{c}\text { FOV } \\
(\mathrm{deg})\end{array}$ & $\begin{array}{c}\mathrm{A} \Omega \\
(\mathrm{m} \mathrm{deg})^{2}\end{array}$ & Mode & $\begin{array}{c}\text { "Sensitivity" } \\
60 \text { sec } 5 \sigma\end{array}$ \\
\hline ConCam & 0.004 & 180 & 0.4 & All sky continuous & 6 \\
WASP & 0.1 & 15 & 1.7 & Triggered \& Partial Survey & 15 \\
ROTSE-III & 0.45 & 2 & 0.6 & Triggered \& Partial survey & 18 \\
Raptor & 0.07 & 35 & 4.7 & Triggered & 16 \\
ASAS & 0.10 & 3 & 0.07 & All sky, daily & 14 \\
HAT & 0.1 & 8 & 0.5 & Precision partial survey & 15 \\
LINEAR & 1.0 & 1.4 & 1.5 & Ecliptic & 19 \\
SDSS & 2.5 & 1.5 & 11 & Partial survey & 22 \\
Palomar/QUEST & 1.2 & 4 & 18 & Multicolor partial survey & 22 \\
${ }^{*}$ VST & 2.6 & 1 & 5.3 & Allocated time & 22 \\
${ }^{*}$ PSS-1 & 1.8 & 2.6 & 23 & All-sky Survey & 22 \\
${ }^{*}$ PS-4 & four $\mathrm{x} 1.8$ & 3 ea & 91 & All-sky survey & 23 \\
${ }^{*}$ LSST & 8.5 & 3 & 510 & "All-sky" Survey & 24 \\
KAIT & 0.8 & 0.1 & 0.005 & Nearby galaxies & 19
\end{tabular}

the Galactic center for light in the $\mathrm{K}$ band $(\lambda=2.2 \mu \mathrm{m})$ vs. light in the $\mathrm{V}$ band $(0.55$ $\mu \mathrm{m})$.

\section{Existing optical detections are already interesting}

Of the supernova detections listed on the CfA web site (http://cfa-www.harvard.edu/iau/lists/RecentSupernovae.html) I found 762 that coincided with the S5 science run of LIGO and VIRGO. Of these, 411 were spectroscopically confirmed as core collapse (type II) SNe, and 89 of these were in catalogued host galaxies. I then used SIMBAD to query the list of named galaxies and obtain their catalogued redshifts. Using an estimate of $\mathrm{H}_{o}=75 \mathrm{~km} / \mathrm{sec}$ per Mpc I generated an approximate distance to each of these supernovae. The closest of the type II SNe from S5, in named host galaxies, are listed in Table 3 .

While the current estimates of gravitational radiation from these particular objects do not lead us to expect a detectable signal, it certainly makes sense to look. In fact, 
Table 2. A comparison of extinction effects in the $\mathrm{V}$ and $\mathrm{K}$ bands. The columns list extinction (in magnitudes) in $\mathrm{V}$ and $\mathrm{K}$ in the first two columns, assuming a canonical relationship of $A_{K}=0.112 * A_{V}$. The third and fourth columns present the expected peak magnitude for a heavily extincted type II supernova at a distance of $10 \mathrm{kpc}$, essentially on the other side of the Galaxy. Working thorough a region with $A_{V}>20$ in the optical is very hard, while in the $\mathrm{K}$ band it is entirely tractable to imagine detecting a typical type II SN through 150 magnitudes of $V$ band attenuation, using a 1 meter class telescope!

\begin{tabular}{rrrr}
$A_{V}$ & $A_{K}$ & $V$ & $K$ \\
\hline 0 & 0 & -3 & -3 \\
5 & 0.6 & 2 & -2.4 \\
10 & 1.1 & 7 & -1.9 \\
15 & 1.7 & 12 & -1.3 \\
20 & 2.2 & 17 & -0.8 \\
50 & 5.5 & 47 & 2.6 \\
100 & 11.1 & 97 & 8.1 \\
150 & 16.7 & 147 & 13.7
\end{tabular}

Table 3. Three relatively close type II supernovae that coincided with LIGO run S5. The columns list the IAUC supernova designation, its host galaxy, and an estimate of the distance.The progenitor characteristics are from Li et al. (2007). Since these were drawn from only 89 of the $762 \mathrm{SNe}$ that occurred during run S5, there are likely others that were closer.

\begin{tabular}{llll} 
IAUC SN & Host & D (Mpc) & Progenitor notes \\
\hline 2006bp & NGC 3953 & 14 & \\
2006my & NGC 4651 & 23 & 7-15 solar mass star \\
2006ov & M61 & 21 & 12-20 solar mass star
\end{tabular}

it seems to me a good idea to obtain a distance estimate to every galaxy that hosted a supernova during run S5, and to search for evidence of strain signals that might be associated with these events. The host galaxy redshifts can often be extracted from the same spectra used to determine the SN type.

\section{Blind Spot \#1: The Milky Way plane}

Except for the all-sky camera systems described above and some of the microlensing projects, most sky survey programs intentionally avoid observing the plane of the Milky Way. This means that for sources fainter than about $6^{\text {th }}$ magnitude, we are currently blind to transient sources that lie close to the Galactic plane! This is of course precisely where we'd expect to find supernovae in our own Galaxy. The historical supernovae in the Milky Way occurred (Richmond 2007) at Galactic latitudes 
of $b=0.1,0.8,32,-6.5,2.7,1.2$ and 7.6. degrees. Now that we realize objects like the Sagittarius galaxy lie behind the Galactic center, a deeper Galactic plane monitoring program seems well motivated. We would also presently miss transient events in external galaxies that lie in the "zone of avoidance" behind the Galactic disk. I re-iterate that frame subtraction analysis can be used to suppress the light from static sources. If we focus on variability detection then the deleterious effects of looking through the Galactic disk are added sky background, and extinction. Augmenting the all-sky optical cameras like ConCam with a wide field imager at $\lambda \sim 2 \mu m$ would be very inexpensive.

\section{Blind Spot \#2: Really bright transients}

The extreme disparity between the optical and gravitational signal strenghts suggests that we pay attention to being able to measure (in multiple filters) the transient OIR flux from bright sources. For a bright core collapse supernova within the local group, the professional optical and IR astronomy communities are not in a position to exploit well-calibrated instruments to observe a naked eye object. As the frontier of optical astronomy has shifted to ever fainter sources, smaller telescopes have been relegated to lower priority. The SN 1987A experience suggests that the astronomical community will adapt, if necessary with neutral density filters duct taped into the instrument, but the time lost to scrambling will lose precious early light curve data. Local supernovae are very rare, and we are not currently prepared to detect and follow one. It's the prospect of detecting optical and gravitational radiation plus neutrinos that makes these bright objects so special, and we should prepare accordingly.

\section{Opportunities, and suggested next steps}

A summary of the opportunities and obvious next steps includes:

- Implement real-time frame subtraction analysis of all-sky camera data.

- Assess merits of optical vs. IR Galactic plane monitoring program.

- Build and operate optimal Galactic plane monitoring system.

- Calculate optical and IR variability that accompanies coalescence events.

- Assess and if needed augment optical all-sky monitoring projects.

- Search for gravitational wave signals associated with all known SNe, and all unclassified optical transients.

- Obtain distances and estimates of $t_{0}$ for all SNe that coincide with run S5.

- Establish stronger collaborations across the optical, IR, gravitational and neutrino communities. 


\section{Acknowledgments, and a dedication}

I am grateful to the organizers of the $12^{\text {th }}$ Gravitational Wave Data Analysis Workshop, held in Cambridge MA in Dec 2007, for the invitation to attend and to present this perspective. My interest in this topic was re-kindled during a visit to Syracuse University, and I thank Duncan Brown and Peter Saulson for interesting conversations during that trip. It was a pleasure to speak with a long-standing friend, Carl Akerlof, about the ROTSE system. I also am grateful for conversations with Avi Loeb and Bence Kocsis, at the Center for Astrophysics at Harvard. I especially thank B. Kocsis and D. Brown for their helpful comments on an early draft of this manuscript. Cullen Blake brought the LIGO/GRB preprint by to my attention. I dedicate this paper to the memory of Bodhan Paczynski, who was a tireless advocate for continual optical monitoring of the sky.

\section{References}

Armitage, P J \& Natarajan, P 2002 ApJ 567, L9.

Barris, B., Tonry, J.L., Novicki, M.C., Wood-Vasey, W.M 2005 AJ 130, 2272.

Becker, A.C. et al. 2004 ApJ 611, 418.

Belczynski, K et al. 2002, ApJ 572, 407.

Bogdanovic, T, Smith, B D, Siguresson, S and Eracleous, M 2007 arXiv:0708.0414, to appear in ApJ Supp.

Clarkson, C A et al. 2003 ApJ 613, 492

Deffayet, C and Menou, K et al. 2007 arXiv:0709.0003

Dotti, M et al. 2006 MNRAS 372, 869.

Filippenko, A. et al. 2001 ASP Conference Series 246121

Grossan, B et al. 1999 AJ, 118, 705

Holz, D \& Hughes, S A 2005 ApJ 629, 15.

Kocsis, B et al. 2006 ApJ 637, 27

LIGO Collaboration \& Hurley, K. 2007, arXiv:0711.1163

Lucas, P W et al. 2007 MNRAS (submitted), arXiv:0712.0100

Milosavljevic, M \& Phinney, S 2006 ApJ 622, L93.

Ott, C et al. 2006 PRL 96, 1102.

Paczynski, B 2006 PASP 118, 1621

Shamir, L and Nemiroff, R.J. 2005 PASP 117, 972.

Pollacco D K et al. 2006 PASP 118, 1407

Richardson, D et al. 2002 AJ, 123, 745.

Richmond, M. 2007, www.tass-survey.org/richmond/answers/historical.html

Rosswog, S 2005 ApJ 634, 1202 
Sessar, B. et al. $2007 A J, \mathbf{1 3 4}, 2236$.

Smith, N et al. 2007 ApJ 666, 1116

Stokes, G.H. et al. 1998 BAAS 30, 1042.

Sylvestre, J 2003 ApJ, 591, 1152

Miknaitis, G. et al. (the ESSENCE Collaboration) 2007 ApJ 666, 674.

Wozniak, P.R. et al. 2005 ApJ 627, L13.

Yost, S.A. et al. 2006 Astronomische Nachrichten 327, 803 